OPEN ACCESS

Edited by:

Martin Burtscher,

University of Innsbruck, Austria

Reviewed by:

Alexander Christoph Stahn,

University of Pennsylvania,

United States

Ulrich Limper,

Krankenhaus Köln-Merheim, Klinikum der Universität Witten/Herdecke,

Germany

*Correspondence: Kouhyar Tavakolian

kouhyar@und.edu

Specialty section:

This article was submitted to Environmental, Aviation and Space

Physiology,

a section of the journal

Frontiers in Physiology

Received: 11 February 2018

Accepted: 23 May 2018

Published: 25 June 2018

Citation:

Verma AK, Xu D, Bruner M, Garg A,

Goswami N, Blaber AP and

Tavakolian K (2018) Comparison

of Autonomic Control of Blood

Pressure During Standing and Artificial Gravity Induced via

Short-Arm Human Centrifuge.

Front. Physiol. 9:712.

doi: 10.3389/fphys.2018.00712

\section{Comparison of Autonomic Control of Blood Pressure During Standing and Artificial Gravity Induced via Short-Arm Human Centrifuge}

\author{
Ajay K. Verma ${ }^{1}$, Da Xu², Michelle Bruner ${ }^{2}$, Amanmeet Garg ${ }^{3}$, Nandu Goswami ${ }^{4}$, \\ Andrew P. Blaber ${ }^{1,2}$ and Kouhyar Tavakolian ${ }^{1,2 *}$
}

${ }^{1}$ Department of Electrical Engineering, University of North Dakota, Grand Forks, ND, United States, ${ }^{2}$ Department of Biomedical Physiology and Kinesiology, Simon Fraser University, Burnaby, BC, Canada, ${ }^{3}$ Department of Engineering Science, Simon Fraser University, Burnaby, BC, Canada, ${ }^{4}$ Physiology Division, Otto Loewi Research Center for Vascular Biology, Immunology and Inflammation, Medical University of Graz, Graz, Austria

Autonomic control of blood pressure is essential toward maintenance of cerebral perfusion during standing, failure of which could lead to fainting. Long-term exposure to microgravity deteriorates autonomic control of blood pressure. Consequently, astronauts experience orthostatic intolerance on their return to gravitational environment. Ground-based studies suggest sporadic training in artificial hypergravity can mitigate spaceflight deconditioning. In this regard, short-arm human centrifuge (SAHC), capable of creating artificial hypergravity of different g-loads, provides an auspicious training tool. Here, we compare autonomic control of blood pressure during centrifugation creating 1-g and 2-g at feet with standing in natural gravity. Continuous blood pressure was acquired simultaneously from 13 healthy participants during supine baseline, standing, supine recovery, centrifugation of 1-g, and 2-g, from which heart rate $(\mathrm{RR})$ and systolic blood pressure (SBP) were derived. The autonomic blood pressure regulation was assessed via spectral analysis of RR and SBP, spontaneous baroreflex sensitivity, and non-linear heart rate and blood pressure causality (RR $\leftrightarrow S B P$ ). While majority of these blood pressure regulatory indices were significantly different ( $p<0.05)$ during standing and 2-g centrifugation compared to baseline, no change $(p>0.05)$ was observed in the same indices during 2-g centrifugation compared to standing. The findings of the study highlight the capability of artificial gravity (2-g at feet) created via SAHC toward evoking blood pressure regulatory controls analogous to standing, therefore, a potential utility toward mitigating deleterious effects of microgravity on cardiovascular performance and minimizing post-flight orthostatic intolerance in astronauts.

Keywords: microgravity, artificial gravity, blood pressure regulation, orthostatic intolerance, astronauts, shortarm human centrifuge, long duration spaceflight, cardiovascular deconditioning 


\section{INTRODUCTION}

Right from birth, humans experience gravity, which pulls the human body toward the earth with a force equivalent to the product of the body mass and the gravitational acceleration, i.e., $9.8 \mathrm{~m} / \mathrm{s}^{2}$ (Antonutto and di Prampero, 2003). Therefore, quintessential physiological performance is highly dependent on gravity (Blaber et al., 2013b; Tanaka et al., 2016). Physiological adaptation, a result of long-term microgravity exposure, can cause changes in physiological functions (Lambertz et al., 2003; Hallgren et al., 2015; Otsuka et al., 2015; Morita et al., 2016; Harris et al., 2017). Of such, cardiovascular adaptation to microgravity can have detrimental effects on the autonomic control of blood pressure upon return to gravitational environment (Hargens et al., 2013; Tanaka et al., 2016). Orthostatic intolerance, an inability to regulate blood pressure on assuming upright stance (Robertson, 1999; Lambert and Lambert, 2014), is commonly experienced by astronauts on their return to Earth after longduration spaceflight (Buckey et al., 1996; Lee et al., 2015). The success of envisioned Mars exploration would entail frequent long-duration spaceflight in the future (Manzey, 2004; Baisden et al., 2008; Clément et al., 2016). To this end, profound investigation of potential countermeasures is warranted to mitigate the adverse effects of microgravity on physiological performance to facilitate healthy life for astronauts on their return to Earth (Clément et al., 2016).

The transition of posture, from supine to standing, reduces the blood pressure at the brain level $(\sim 70 \mathrm{mmHg})$ and increases the blood pressure at the feet level $(\sim 200 \mathrm{mmHg})$, as a consequence of gravity (Hargens and Richardson, 2009; Hargens et al., 2013). Central blood volume, owing to gravity induced hydrostatic pressure gradient, is displaced below heart level and venous return and preload are reduced challenging blood pressure equilibrium. Regulating blood pressure is imperative for the sustainability of prolonged and stable upright stance. During physiological state of standing, blood pressure is regulated via reduced afferent discharge of the baroreceptor impulses localized in the carotid sinus and the aortic arch, leading to increased heart rate and systemic vascular resistance as a consequence of vagal withdrawal and sympathetic nerve activation (Smith et al., 1994; Heesch, 1999).

Therefore, to cope with the effects of orthostatic challenge on physiological performance, autonomic, baroreceptor, and vasomotor controls play a consequential role to various degrees. Enfeebled or impaired blood pressure regulatory controls owing to long-term microgravity exposure could lead to an abrupt decline in arterial blood pressure on assuming upright stance resulting in reduced cerebral perfusion (Olufsen et al., 2004, 2005; Shaw and Claydon, 2014). Thus, impaired autonomic control has an ominous effect on the individual's ability to maintain standing (Antonutto and di Prampero, 2003; Hargens et al., 2013; Tanaka et al., 2016).

Ground-based experiments, performed to comprehend physiological response to microgravity simulated via bed rest immobilization (LeBlanc et al., 1988; Jeong et al., 2013; Norsk, 2014) and potential countermeasures such as exercise training, lower-body negative pressure, and artificial gravity (Goswami et al., 2008; Blaber et al., 2013a; Hargens et al., 2013), have concluded an intermittent exposure to artificial hypergravity as an important factor toward improving orthostatic tolerance (Evans et al., 2004; Stenger et al., 2007, 2012; Hargens et al., 2013). The short-arm human centrifuge (SAHC), in this regard, can serve as a promising training tool (Evans et al., 2004; Frett et al., 2014; Clément et al., 2015). The feasibility of short-arm centrifuge to be a part of a long duration spaceflight, owing to compact modern design, has opened new avenues toward minimizing the severity of microgravity-induced systemic deconditioning (Bukley et al., 2007; Zander et al., 2013; Frett et al., 2014; Diaz et al., 2015).

In the previous work from our group (Goswami et al., 2015a), we demonstrated the response of cardiovascular and the cerebrovascular system during 2-g centrifugation at feet to be analogous to orthostatic challenge exerted by standing in a natural gravity. However, the autonomic blood pressure regulatory controls during centrifuge induced artificial gravity in relation to standing in natural gravity remain to be generalized. In the current analysis, we extend our previous work to a comparison of the response of autonomic control of blood pressure during artificial gravity induced via SAHC in relation to standing. The autonomic control of blood pressure was assessed via causal heart rate-blood pressure interaction, spontaneous baroreflex sensitivity, and spectral analysis of SBP and RR time series.

\section{MATERIALS AND METHODS}

\section{Experimental Protocol and Data Acquisition}

The detailed experimental protocol has been explained in the previous work from our group (Goswami et al., 2015a). Here, we briefly outline the experimental protocol with respect to the current research. All participants were pre-screened for physical and medical status. None of the participants had a prior history of cardiovascular, neurological, and musculoskeletal diseases or vasovagal syncope. Twelve hours prior to experimentation, all participants were required to refrain from alcohol, caffeine, and any medication.

In the centrifuge, the participant was strapped with their head near the center of 2.8 -m radius centrifuge and feet outwards. The participant remained supine (Baseline) in the centrifuge for 20 min of baseline recording. After completion of baseline, the participant was transitioned with assistance into the standing position for $5 \mathrm{~min}$ (stand test). After the stand test, the participant lay supine (recovery) in the centrifuge for another $15 \mathrm{~min}$, after which the centrifuge was ramped up to a rate that applied 1-g at feet $(0.22 \mathrm{~g}$ at Middle Cerebral Artery and $0.39 \mathrm{~g}$ at heart) for $5 \mathrm{~min}$ and then increased to $2-\mathrm{g}$ at feet $(0.44 \mathrm{~g}$ at Middle Cerebral Artery and $0.75 \mathrm{~g}$ at the heart) for $5 \mathrm{~min}$. Following 5 min of centrifugation at 2-g, the centrifuge rotation was slowed and halted in $30 \mathrm{~s}$.

Data was acquired from 13 participants (age: $28.08 \pm 8.4$ year, height: $172 \pm 6.9 \mathrm{~cm}$, weight: $67.6 \pm 10.5 \mathrm{~kg}$, six females). The detailed demographic information is provided in Table $\mathbf{1}$ 
TABLE 1 | Detailed demographic information of study participants.

\begin{tabular}{ccccl}
\hline Participant \# & Age & Height & Weight & Gender \\
\hline 1 & 23 & 172 & 73 & Male \\
2 & 31 & 165 & 55 & Female \\
3 & 24 & 164 & 56 & Female \\
4 & 25 & 169 & 62 & Female \\
5 & 25 & 176 & 75 & Male \\
6 & 35 & 175 & 76 & Male \\
7 & 23 & 165 & 53 & Female \\
8 & 29 & 175 & 79 & Male \\
9 & 24 & 174 & 55 & Female \\
10 & 35 & 189 & 84 & Male \\
11 & 39 & 178 & 73 & Male \\
12 & 37 & 166 & 72 & Male \\
13 & 32 & 169 & 68 & Female \\
\hline
\end{tabular}

Continuous blood pressure was acquired from noninvasive finger photoplethysmography cuff (Portapress, FMS, Netherlands) using NI data acquisition (National Instruments, Inc., Austin, TX, United States) system at a sampling rate of $1000 \mathrm{~Hz}$. Ethics approval for experimentation was obtained from the University of Toulouse. Experimentation complied with rules and regulations set forth by the research ethics board of the University of Toulouse. Written and informed consent form for participation was obtained from each participant prior to any experimentation.

\section{Short-Arm Human Centrifuge}

Short-arm human centrifuge is a training tool capable of creating artificial gravity of different g-load (Zander et al., 2013; Clément et al., 2015). Briefly, the head of participant is aligned close to the center of centrifuge rotation, while the feet are directed outwards from the center of rotation. In this orientation, the g-load is distributed linearly in a head-to-toe axis, i.e., the g-load at the feet is hypothesized to be proportional to the rotational speed of the centrifuge, while at the head the g-load is closer to zero. Although this does not simulate the typical hydrostatic difference created by standing in a natural gravity, it does generate similar physical stressors in the footward direction. The relation between artificial gravity created at feet on Earth and rotation of centrifuge can be described as; $\mathrm{CF}=\frac{\mathrm{rx} \omega^{2}}{\mathrm{~g}}$. Where CF is a centrifugal force, $r$ is the radius at feet, $\omega$ is the rotational speed, and $g$ is the Earths gravitational acceleration (Clément and Pavy-Le Traon, 2004). The centrifuge facility at Institute for Space Medicine and Physiology (MEDES), Toulouse, France was used in this research.

\section{Data Processing}

Diastolic nadirs of each beat were first detected from the continuous blood pressure waveform to obtain diastolic blood pressure (DBP). The R-R time interval was obtained as the duration between two adjacent diastolic nadirs. Systolic blood pressure (SBP) was obtained as the maximum blood pressure between two adjacent diastolic nadirs. Beat-by-beat mean arterial pressure (MAP) was derived from blood pressure waveform as a mean value between two adjacent DBP locations. 5-min of data from each experimental condition was considered for analysis.

RR interval and SBP time series were interpolated using spline interpolation to generate an evenly sampled signal and resampled to $2 \mathrm{~Hz}$ with zero mean before conducting the spectral analysis. The Welch power spectral density (PSD) of RR and SBP was calculated in very low frequency (VLF, 0-0.04 Hz), low frequency (LF, 0.04-0.15), and high frequency (HF, 0.15$0.4 \mathrm{~Hz}$ ) bands. There upon, the SBP power distributed (P) in the respective bands were normalized as $\mathrm{VLF}_{\mathrm{nu}}=\mathrm{P}_{\mathrm{VLF}} \div$ Total Power, $\mathrm{LF}_{\mathrm{nu}}=\mathrm{P}_{\mathrm{LF}} \div$ Total Power, and $\mathrm{HF}_{\mathrm{nu}}=\mathrm{P}_{\mathrm{HF}} \div$ Total Power, in case of RR signal the normalized power in only LF and $\mathrm{HF}$ frequency band was calculated as $\mathrm{LF}_{\mathrm{nu}}=\mathrm{P}_{\mathrm{LF}} \div$ (Total Power- $\left.\mathrm{P}_{\mathrm{VLF}}\right)$ and $\mathrm{HF}_{\mathrm{nu}}=\mathrm{P}_{\mathrm{HF}} \div\left(\right.$ Total Power- $\left.\mathrm{P}_{\mathrm{VLF}}\right)$ where Total Power $=\mathrm{P}_{\mathrm{VLF}}+\mathrm{P}_{\mathrm{LF}}+\mathrm{P}_{\mathrm{HF}}$ in accordance with recommendation in the literature (Malik et al., 1996). The PSD was computed with a Hamming window of size 128 samples and 50\% overlap.

The arterial baroreflex sensitivity was calculated using sequence method (Bertinieri et al., 1988; Blaber et al., 1995) by using CardioSeries computer software V2.4 ${ }^{1}$ similar to other research in the literature (Durand et al., 2015; Silva et al., 2015). Beat-to-beat RR intervals and SBP were input to the software, search for a sequence of at least three consecutive beats in which increase in SBP was followed by an increase in RR intervals (up slope) and decrease in SBP followed by a decrease in RR intervals (down slope) with a correlation greater than 0.8 was considered. The slope of linear regression between SBP and RR intervals was considered as a marker of spontaneous BRS.

The strength of closed loop heart rate-blood pressure interaction $(\mathrm{RR} \leftrightarrow \mathrm{SBP})$, signifying the feedforward (nonbaroreflex) and feedback (baroreflex) controls of blood pressure was obtained using convergent cross mapping (CCM) similar to our previous work (Verma et al., 2017b; Xu et al., 2017). Prior to causality analysis, the evenly sampled continuous RR and SBP signals were resampled to $10 \mathrm{~Hz}$. Mathematical details of the methodology are provided in the supplementary material of Sugihara et al. (2012) and in a book on time series analysis by Mccracken (2016).

\section{Statistical Analysis}

Test for normal distribution of the mean of different variables studied in this research was conducted using Shapiro-Wilk test (SPSS, IBM Corporation, Armonk, NY, United States). A multiple comparison test was conducted using repeated measure of ANOVA (for normally distributed data) or Friedman test (data failed normality) followed by post hoc analysis using TukeyHSD method to account for the significance of changes in the cardiovascular parameters and blood pressure regulatory indices during different experimental conditions. The test of significance was conducted using a statistical toolbox of MATLAB (The Mathworks, Inc., Natick, MA, United States). The test results at $\alpha=0.05$ were considered significant. All tabular data in the article are presented as mean \pm SD unless mentioned otherwise.

\footnotetext{
${ }^{1} \mathrm{http}: / /$ www.danielpenteado.com
} 


\section{RESULTS}

Test of normality resulted in data exhibiting mixed behavior given the limited sample size, therefore, Friedman test followed by post hoc analysis using Tukey-HSD method was conducted to account for difference exerted by different experimental conditions on the cardiovascular parameters as well as on the blood pressure regulatory indices. Table 2 summarizes the behavior of cardiovascular parameters during baseline, standing, recovery, centrifugation of 1-g, and 2-g. Stand test or application of centrifugation inflicted no change in SBP $(p=0.13)$, DBP $(p=0.27)$, or MAP $(p=0.28)$. RR intervals reduced significantly during standing $(p<0.001)$ and 2-g $(p<0.001)$ compared to supine. Additionally, RR intervals reduced significantly during standing $(p<0.001)$ and $2-\mathrm{g}(p<0.001)$ compared to recovery as well as during $2-\mathrm{g}(p=0.02)$ compared to 1 -g. No change in RR intervals was obtained during recovery $(p=0.99)$ and 1-g $(p=0.09)$ compared to baseline. Furthermore, no change $(p=0.57)$ in RR intervals was observed between standing and 2-g. Figure 1 shows an example of RR intervals and blood pressure dynamics in response to different experimental conditions.

Figure 2 summarizes the normalized spectral power distribution in the VLF, LF, and HF bands of SBP and LF, HF, and LF/HF ratio of RR intervals. The spectral power distribution in the respective frequency bands of SBP and RR in absolute and normalized units is summarized in Table 3. Table 4 lists the post hoc comparison $p$-value between experimental conditions for respective frequency bands. Significant change was observed in low-frequency SBP power both in normalized and absolute units during standing $(p=0.04$ and $p=0.01)$ and $2-\mathrm{g}(p<0.001$ and $p<0.001)$ compared to baseline. Moreover, the lowfrequency SBP power (both in normalized and absolute power) was significantly different during standing and 2-g compared to recovery. In addition, the $\mathrm{SBP}_{\mathrm{LF}}$ and $\mathrm{SBP}_{\mathrm{HF}}$ in absolute power were observed to be significantly different $(p<0.05)$ at 2 -g compared to 1 -g (Table 3). No difference $(p>0.10)$ was the behavior of such variable at 2-g compared to standing (Table 4).

The spectral power (n.u.) in the LF and HF frequency bands of RR intervals increased $(p=0.007)$ and decreased $(p=0.007)$ significantly only at 2 -g compared to baseline (Table 4 ). The LF/HF ratio increased significantly at $2-\mathrm{g}(p=0.007)$ compared to baseline. Moreover, the three metrics were significantly different $(p<0.05)$ during standing and 2 -g compared to recovery (Table 3). In terms of absolute power no change $(p=0.87)$ was observed in the $R R_{L F}$, while $R_{H F}$ decreased significantly $(p<0.05)$ during standing and 2-g compared to baseline and recovery (Table 3$)$. No difference $(p>0.10)$ in the dynamics of RR spectral power was obtained at $2-\mathrm{g}$ compared to standing (Table 4).

Baroreflex sensitivity decreased (both up and down slope) significantly during standing (up slope, $p=0.007$ and down

TABLE 2 | Values (mean \pm SD) of cardiovascular parameters during different experimental conditions.

\begin{tabular}{|c|c|c|c|c|c|}
\hline Parameters & Baseline & Stand & Recovery & $1-g$ & $2-g$ \\
\hline $\mathrm{SBP}(\mathrm{mmHg})$ & $110 \pm 15$ & $122 \pm 21$ & $118 \pm 13$ & $119 \pm 16$ & $114 \pm 21$ \\
\hline MAP (mmHg) & $74 \pm 11$ & $80 \pm 18$ & $78 \pm 12$ & $76 \pm 12$ & $74 \pm 17$ \\
\hline
\end{tabular}

Results were considered significant at $\alpha=0.05 .{ }^{*}$ Represents significant difference from baseline. ${ }^{\dagger}$ Represents significant difference from recovery. ${ }^{\ddagger}$ Represents significant difference from $1-g$.
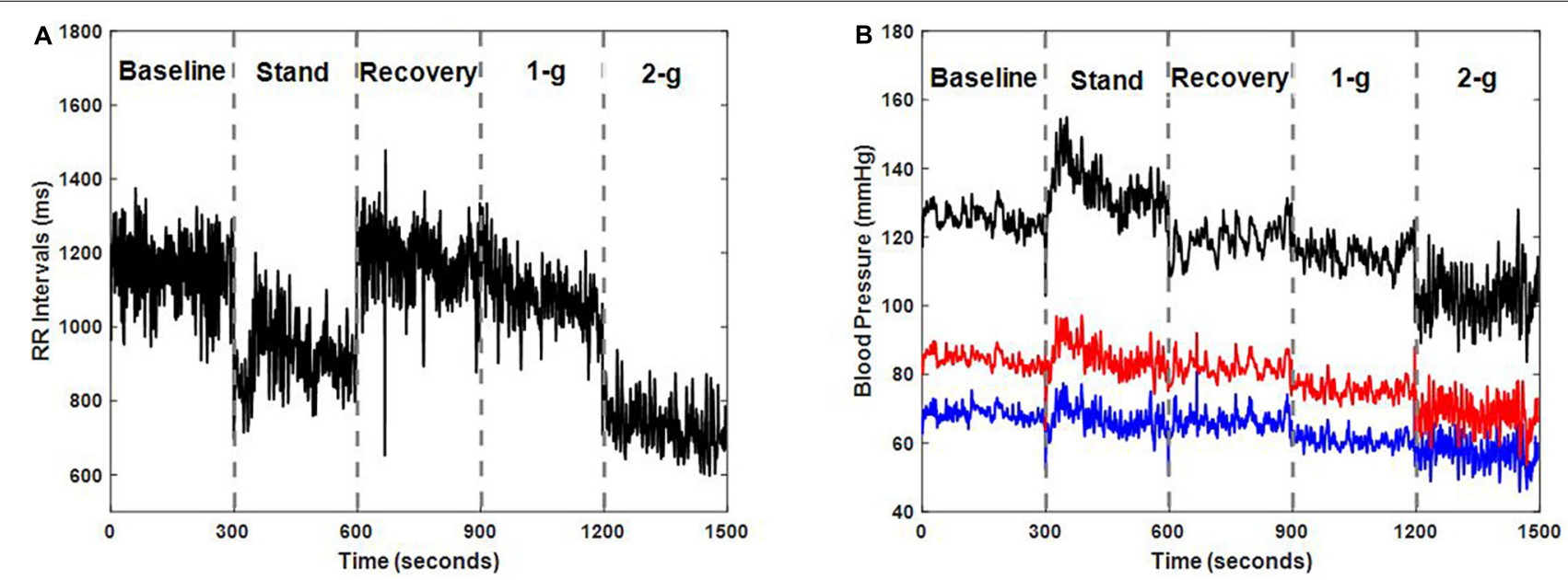

FIGURE 1 | Dynamics of RR intervals (A) and blood pressure (B) i.e., systolic blood pressure (SBP) (black), diastolic blood pressure (DBP) (blue), and mean arterial pressure (MAP) (red) in response to different experimental conditions for one participant (male, age: 35 years, height: $175 \mathrm{~cm}$, weight: $76 \mathrm{~kg}$ ). 

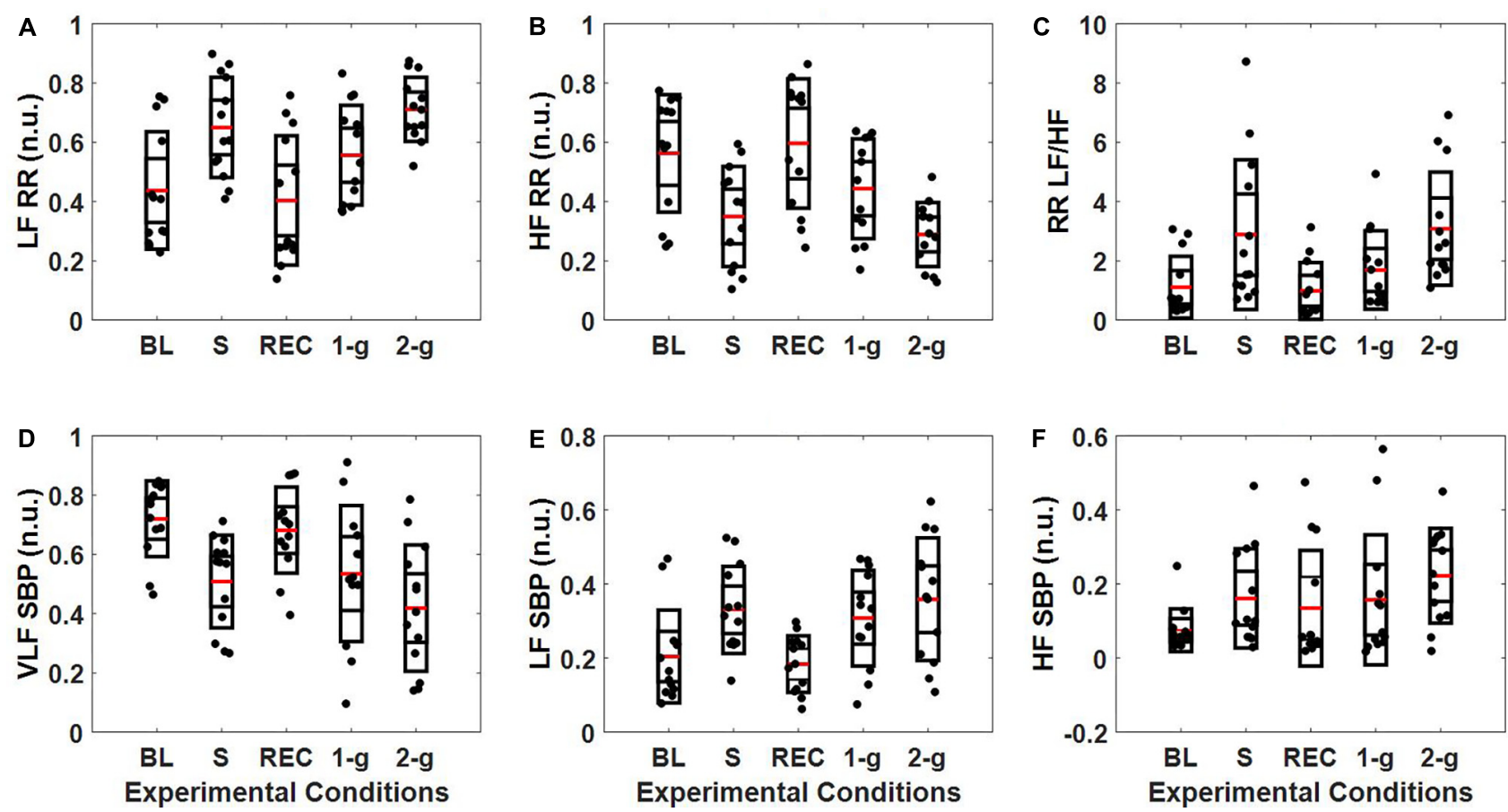

FIGURE 2 | Distribution of SBP and RR intervals spectral power (n.u.). The figure details RR (A-C) and SBP (D-F) spectral power distribution in the VLF (0-0.04 Hz), LF (0.04-0.15 Hz, and HF (0.15-0.4 Hz) bands during baseline (BL), stand (S), recovery (REC), 1-g, and 2-g experimental protocol.

TABLE 3 | Values (mean \pm SD) of blood pressure regulatory indices in response to different experimental conditions.

\begin{tabular}{|c|c|c|c|c|c|}
\hline Blood pressure regulatory indices & Baseline & Stand & Recovery & $1-g$ & $2-g$ \\
\hline $\operatorname{SBP}_{V L F}($ n.u.) & $0.72 \pm 0.13$ & $0.51 \pm 0.16$ & $0.68 \pm 0.15$ & $0.54 \pm 0.23$ & $0.42 \pm 0.21^{* \dagger}$ \\
\hline $\mathrm{SBP}_{\mathrm{LF}}$ (n.u.) & $0.20 \pm 0.13$ & $0.33 \pm 0.12^{* \dagger}$ & $0.18 \pm 0.08$ & $0.31 \pm 0.11$ & $0.35 \pm 0.17^{* \dagger}$ \\
\hline $\mathrm{SBP}_{\mathrm{HF}}$ (n.u.) & $0.08 \pm 0.06$ & $0.16 \pm 0.13$ & $0.13 \pm 0.16$ & $0.16 \pm 0.18$ & $0.22 \pm 0.13$ \\
\hline $\mathrm{SBP}_{\mathrm{VLF}}\left(\mathrm{mmHg}^{2}\right)$ & $22.02 \pm 11.58$ & $21.60 \pm 11.78$ & $22.43 \pm 20.63$ & $16.07 \pm 16.14$ & $29.67 \pm 32.84$ \\
\hline $\mathrm{SBP}_{\mathrm{LF}}\left(\mathrm{mmHg} \mathrm{g}^{2}\right)$ & $5.80 \pm 3.95$ & $14.36 \pm 8.44^{* \dagger}$ & $5.19 \pm 4.05$ & $6.91 \pm 4.86$ & $20.93 \pm 13.02^{* \dagger \ddagger}$ \\
\hline $\mathrm{SBP}_{\mathrm{HF}}\left(\mathrm{mmHg}{ }^{2}\right)$ & $1.89 \pm 1.01$ & $6.41 \pm 6.49$ & $1.94 \pm 1.36$ & $2.37 \pm 1.97$ & $11.41 \pm 8.44^{* \dagger \ddagger}$ \\
\hline$R R_{L F}$ (n.u.) & $0.44 \pm 0.20$ & $0.65 \pm 0.17^{\dagger}$ & $0.40 \pm 0.22$ & $0.56 \pm 0.17$ & $0.71 \pm 0.11^{* \dagger}$ \\
\hline $\mathrm{RR}_{\mathrm{HF}}$ (n.u.) & $0.56 \pm 0.20$ & $0.35 \pm 0.17^{\dagger}$ & $0.60 \pm 0.22$ & $0.44 \pm 0.17$ & $0.29 \pm 0.11^{* \dagger}$ \\
\hline $\mathrm{RR}_{\mathrm{LF} / \mathrm{HF}}$ & $1.11 \pm 1.04$ & $2.89 \pm 2.53^{\dagger}$ & $0.99 \pm 0.96$ & $1.69 \pm 1.33$ & $3.08 \pm 1.91^{* \dagger}$ \\
\hline $\mathrm{RR}_{\mathrm{LF}}\left(\right.$ second $^{2}$ ) & $1.45 \pm 1.40$ & $1.25 \pm 1.31$ & $1.25 \pm 1.42$ & $1.50 \pm 2.06$ & $1.23 \pm 1.09$ \\
\hline $\mathrm{RR}_{\mathrm{HF}}\left(\right.$ second $\left.^{2}\right)$ & $2.53 \pm 3.14$ & $1.01 \pm 1.89^{* \dagger}$ & $3.78 \pm 6.43$ & $1.88 \pm 3.53$ & $0.51 \pm 0.60^{* \dagger}$ \\
\hline $\mathrm{BRS}_{\text {upslope }}(\mathrm{ms} / \mathrm{mmHg})$ & $31.18 \pm 20.19$ & $10.40 \pm 4.78^{* \dagger}$ & $30.65 \pm 20.91$ & $20.10 \pm 9.43$ & $7.52 \pm 3.38^{* \dagger \ddagger}$ \\
\hline $\mathrm{BRS}_{\text {downslope }}(\mathrm{ms} / \mathrm{mmHg})$ & $29.31 \pm 18.15$ & $10.85 \pm 6.39^{* \dagger}$ & $27.51 \pm 16.78$ & $21.32 \pm 10.16$ & $6.80 \pm 2.67^{* \dagger \ddagger}$ \\
\hline $\mathrm{RR} \rightarrow \mathrm{SBP}$ & $0.95 \pm 0.03$ & $0.93 \pm 0.03$ & $0.93 \pm 0.03$ & $0.92 \pm 0.05$ & $0.92 \pm 0.04$ \\
\hline $\mathrm{SBP} \rightarrow \mathrm{RR}$ & $0.83 \pm 0.09$ & $0.91 \pm 0.03 *$ & $0.88 \pm 0.07$ & $0.91 \pm 0.04 *$ & $0.89 \pm 0.05$ \\
\hline
\end{tabular}

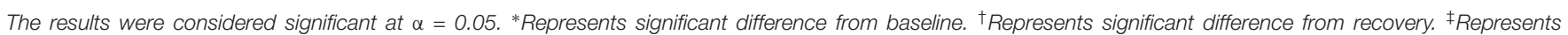
significant difference from $1-\mathrm{g}$.

slope, $p=0.003$ ) and 2-g (up slope, $p<0.001$ and down slope, $p<0.001)$ compared to baseline. Significant decline in BRS was observed during standing (up slope, $p=0.003$ and down slope, $p=0.004$ ) and 2 -g (up slope, $p<0.001$ and down slope, $p<0.001$ ) compared to recovery as well as at 2 -g (up slope, $p=0.04$ and down slope $p=0.005$ ) compared to 1 -g (Table 3 ). No change (up slope, $p=0.79$ and down slope, $p=0.72$ ) in BRS was observed at 2-g compared to standing (Table 4). The distribution of up slope and down slope BRS for the study participants is detailed in Figure 3.

The optimal embedding dimension to perform nonlinear state space reconstruction in CCM was determined via false nearest neighbor algorithm at a delay of 10 samples to account for changes within a heartbeat range. The optimal dimension of reconstruction was determined to be 4 for SBP and RR based on the minimization of false nearest 
TABLE 4 | Comparison of changes in blood pressure regulatory indices inflicted by different experimental conditions.

\begin{tabular}{|c|c|c|c|c|c|c|}
\hline $\begin{array}{l}\text { Variables and conditions } \\
\text { to compare }\end{array}$ & Baseline vs. stand & Baseline vs. recovery & Baseline vs. 1-g & Baseline vs. 2-g & Stand vs. 1-g & Stand vs. $2-g$ \\
\hline $\operatorname{SBP}_{V L F}$ (n.u.) & 0.07 & 0.99 & 0.17 & $0.001^{a}$ & 0.99 & 0.79 \\
\hline $\operatorname{SBP}_{\mathrm{LF}}$ (n.u.) & $0.04^{b}$ & 0.99 & 0.09 & $<0.001^{\mathrm{a}}$ & 0.99 & 0.79 \\
\hline $\mathrm{SBP}_{\mathrm{LF}}$ (abs power) & $0.01^{a}$ & 0.99 & 0.90 & $<0.001^{\mathrm{a}}$ & 0.12 & 0.79 \\
\hline SBP $_{\mathrm{HF}}$ (abs power) & 0.09 & 0.99 & 0.98 & $<0.001^{\mathrm{a}}$ & 0.27 & 0.41 \\
\hline$R R_{L F}$ (n.u.) & 0.13 & 0.79 & 0.85 & $0.007^{a}$ & 0.65 & 0.85 \\
\hline $\mathrm{RR}_{\mathrm{HF}}$ (n.u.) & 0.12 & 0.79 & 0.85 & $0.007^{a}$ & 0.65 & 0.85 \\
\hline $\mathrm{RR}_{\mathrm{LF} / \mathrm{HF}}$ & 0.12 & 0.79 & 0.85 & $0.007^{a}$ & 0.65 & 0.85 \\
\hline $\mathrm{RR}_{\mathrm{HF}}$ (abs power) & $0.02^{b}$ & 0.99 & 0.12 & $0.001^{a}$ & 0.97 & 0.90 \\
\hline $\mathrm{BRS}_{\text {upslope }}$ & $0.007^{a}$ & 0.99 & 0.41 & $<0.001^{a}$ & 0.48 & 0.79 \\
\hline $\mathrm{BRS}_{\text {downslope }}$ & $0.003^{a}$ & 0.99 & 0.65 & $<0.001^{a}$ & 0.16 & 0.72 \\
\hline $\mathrm{SBP} \rightarrow \mathrm{RR}$ & $0.007^{a}$ & 0.21 & $0.04^{b}$ & 0.16 & 0.98 & 0.79 \\
\hline
\end{tabular}

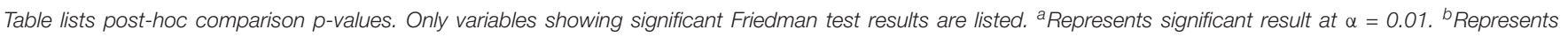
significant result at $\alpha=0.05$.

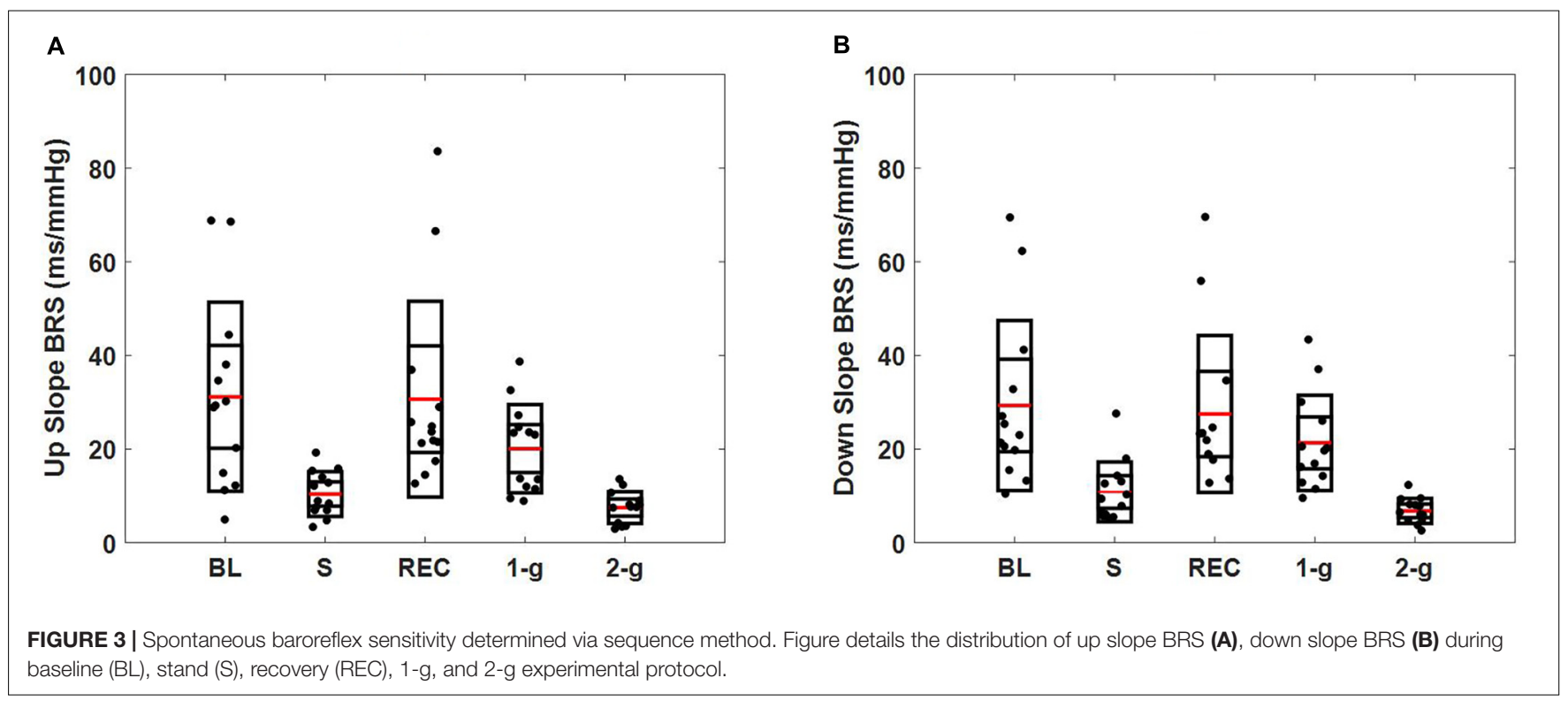

neighbor using CRP toolbox in MATLAB (Kennel and Brown, 1992; Marwan, 2017). Therefore, the $\mathrm{RR} \leftrightarrow \mathrm{SBP}$ causality was computed at an embedding dimension of 4 and delay of 10 samples unless mentioned otherwise. The causal behavior between RR and SBP is detailed in Figure 4.

At supine rest, the non-baroreflex arm of the heart rate and blood pressure interaction was significantly higher than the baroreflex arm. During standing $(p=0.007)$ and $1-\mathrm{g}$ centrifugation $(p=0.04)$, a significant increase in baroreflex arm of the interaction compared to supine was observed, while no change $(p=0.26)$ in the non-baroreflex arm of the interaction was observed during experimental conditions. No change $(p=0.16)$ was observed in the baroreflex arm of the interaction at 2-g compared to baseline. Additionally, no difference between the standing and 2-g centrifugation was observed in the baroreflex arm $(\mathrm{SBP} \rightarrow \mathrm{RR}, p=0.79$ of the heart rate and blood pressure interaction (Table 3).

\section{DISCUSSION}

The current research investigated the capability of centrifugation (1-g and 2-g) at feet to evoke autonomic control of blood pressure analogous to standing. The autonomic blood pressure regulation mechanisms via causal heart rate-blood pressure interaction, spontaneous baroreflex sensitivity, and spectral analysis of SBP and RR were studied. The analysis results ascertained previously contemplated yet undocumented potential of SAHC to evoke autonomic blood pressure control analogous to standing in natural gravity, therefore, a potential utility toward minimizing the adverse effects of long-term microgravity exposure on the cardiovascular performance, hence, minimizing orthostatic intolerance in astronauts upon return to Earth.

The success of future inter-planetary missions to Mars depends on the design of pertinent countermeasures to mitigate the adverse effects of spaceflight deconditioning. The shift of central blood volume above thoracic leading to increased 

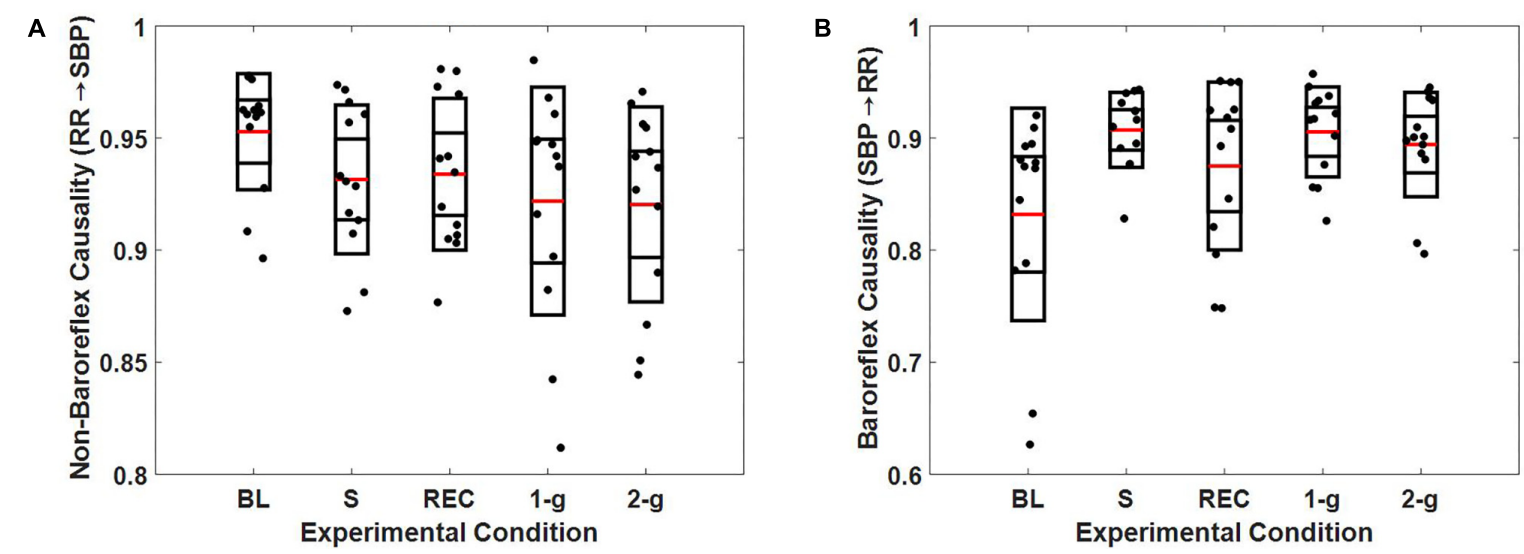

FIGURE 4 | Boxplot representation of non-baroreflex (A) and baroreflex (B) causalities in response to baseline (BL), stand (S), recovery (REC), 1-g, and 2-g experimental protocol.

ventricular filling, stroke volume, and cerebral blood flow is an immediate consequence of physiological adaptation to microgravity. Accordingly, baroreceptor unloading, autonomic sympathetic nerve activity, and the vasomotor control remain vastly inhibited for the duration of spaceflight (White and Averner, 2001; Hargens and Richardson, 2009; Williams et al., 2016). The prolong inhibition of such blood pressure regulatory controls can have an adverse effect on individual's orthostatic tolerance level, which can be analogous to aging and/or pathology (Takamatsu et al., 2016; Goswami, 2017).

Furthermore, decreased blood flow to the peripheral regions, especially to the calf skeletal muscles, render posture muscle group with nutritional scarcity, and is a major contributor toward skeletal muscle atrophy, bone remodeling, and decline in the calf circumference (LeBlanc et al., 1988; Lambertz et al., 2003; Stewart et al., 2004). While assuming upright stance on return to the gravitational environment, the blood pressure regulatory controls such as autonomic blood pressure controls and skeletal muscle pump remains vastly ineffective. Such change in physiological function can lead to excessive pooling of central blood volume in the lower periphery, resulting in cerebral perfusion reduction leading to feeling of dizziness and potentially syncope.

Thus, an external system capable of evoking autonomic control of blood pressure (baroreceptor unloading leading to increased sympathetic, decreased vagal activity, and increased systemic vascular resistance) and simultaneously increasing blood flow to the calf musculature to assure adequate nutritional and metabolic supply can mitigate the deterioration of cardiovascular performance associated with long-term exposure to microgravity.

Exercise training and lower-body negative pressurization have been utilized as a potential countermeasure to spaceflight deconditioning. However, traditionally used aerobic exercise, resistance training, and lower-body suction has been limited in effect due to their inability to challenge multiple physiological systems that are associated with standing (Bukley et al., 2007; Clément et al., 2015; Artiles et al., 2016). Consequently, interest has shifted toward SAHC as a training tool to minimize microgravity-induced physiological deconditioning (Clément and Pavy-Le Traon, 2004). SAHC, given its capability to create artificial gravity and has a potential to produce hydrostatic gradients analogous to standing and evoke multiple physiological systems simultaneously (Zander et al., 2013; Diaz et al., 2015). Achieving desired performance from SAHC, however, is contingent on an ideal choice of g-load. High g-load could initiate early syncopal symptoms, while low g-load could be insufficient to induce strenuous perturbation to hemodynamic homeostasis, and therefore, fails to evoke desired autonomic control of blood pressure. The autonomic control of blood pressure via the conventional approach of arterial baroreflex sensitivity and heart rate variability have been shown to exhibit microgravity or hypergravity induced alteration in the autonomic performance (Yanagida et al., 2014; Fontolliet et al., 2015). In this research, we investigated the capability of $1-\mathrm{g}$ and 2 -g centrifugation toward evoking autonomic controls of blood pressure. Previous research have demonstrated 2-g to be under the safe limit in addition to being strenuous enough to evoke physiological responses analogous to standing (Iwasaki et al., 2001; Goswami et al., 2015a).

We applied external perturbation to the hemodynamic homeostasis via stand test and centrifugation of $1-\mathrm{g}$ and 2-g at feet. A recovery period followed a stand test to minimize its influence on the autonomic behavior during centrifugation. The comparison result of baseline and recovery period is summarized in Tables 1, 4 . The results suggest the dynamics of studied variables were not different between the two conditions, therefore, the residual effects of standing was minimized and not likely to influence the autonomic behavior during centrifugation. The experimental conditions inflicted no change in SBP $(p=0.13)$, DBP $(p=0.27)$, or MAP $(p=0.28)$. This observation suggests that blood pressure was well-regulated during orthostatic challenge evoked via standing and centrifugation by active autonomic control of blood pressure. A significant decline was observed in the power distributed in the HF band of RR during standing $(p=0.02)$ and 2-g $(p=0.001)$ compared to baseline (Table 4), suggesting withdrawal of vagal 
nerve activity; resulting in a shift of sympatho-vagal balance toward sympathetic activity. Also, increase in LF SBP power was observed during standing and 2-g compared to supine (Table 3). Additionally, baroreflex sensitivity (both up slope and down slope) declined during standing and 2-g compared to supine (Table 3), which is the result of decreased vagal activity (Table 4) and increased heart rate or reduced RR intervals (Table 1) contributing toward maintenance of blood pressure equilibrium. While these markers of autonomic controls of blood pressure were significantly different from baseline during standing and 2g, no change $(p>0.10)$ in the autonomic blood pressure control between standing and 2-g was observed (Table 4). Moreover, no change (Table 4) was observed in these indices between baseline and 1 -g. This observation suggests $2-\mathrm{g}$ is more closely related to standing compared to $1-\mathrm{g}$ in terms of stimulating autonomic blood pressure regulatory controls.

While the spectral analysis of SBP and RR time series and baroreflex sensitivity are well-accepted norm to account for the autonomic control of blood pressure, criticism of such approaches in the literature is also prevalent for the inability of the spectral method to account for the non-linearity of underlying physiology and BRS for not able to address the closed loop heart rate and blood pressure interaction (Blaber et al., 1995; Malik et al., 1996; Zhong et al., 2006; Svacinova et al., 2015; Silvani et al., 2017). As such, in addition to traditional measures, the current article studied the non-linear causal heart rate and blood pressure interaction, a closed loop control system. Where the feedforward control signifies the Frank-Starling effect on blood pressure while the feedback control accentuates the baroreflex control of blood pressure. We studied the strength of feedforward (non-baroreflex, RR $\rightarrow$ SBP) and feedback (baroreflex, $\mathrm{SBP} \rightarrow \mathrm{RR}$ ) controls of blood pressure during different experimental conditions (Faes et al., 2013; Javorka et al., 2017).

The results of closed loop heart rate and blood pressure interaction are detailed in Figure 4. No change $(p>0.05)$ was observed in the dynamics of non-baroreflex $(\mathrm{RR} \rightarrow \mathrm{SBP})$ causality during standing or 2-g compared to supine. However, a significant increase was observed in the baroreflex $(\mathrm{SBP} \rightarrow \mathrm{RR})$ causality; both during standing $(p=0.009)$ and 1-g $(p=0.04)$, Table 4. No change $(p=0.16)$ was observed in SBP $\rightarrow \mathrm{RR}$ causality during 2-g compared to baseline. The slight decline in baroreflex causality at 2-g compared to 1-g (Table 3) could be due to the fact that $2-\mathrm{g}$ was more stressful than standing and 1-g evident from certain study variables $\left[\mathrm{SBP}_{\mathrm{VLF}}, \mathrm{SBP}_{\mathrm{HF}}\right.$ (n.u.), $\mathrm{RR}_{\mathrm{HF}}$ (n.u.), and $\mathrm{RR}_{\mathrm{LF}}$ (n.u.), Table 4], which changed only at 2-g compared to baseline. Furthermore, heart rate, $\mathrm{BRS}, \mathrm{SBP}_{\mathrm{LF}}$, and $\mathrm{SBP}_{\mathrm{HF}}$ changed significantly at 2-g compared to 1-g (Table 3), which further hints toward 2-g being more stressful than $1-\mathrm{g}$. The RR intervals reduced at $1-\mathrm{g}(p=0.09)$ albeit not significantly but given limited sample size it does hint toward 1-g being more challenging than baseline. The baroreflex coupling is observed to decline before syncope (Faes et al., 2006), in our study syncope was not evident in the study participants, however, a decline in baroreflex causality indicates if exposed to 2-g centrifugation for an extended period, observation of syncopal symptom is plausible. Additionally, no change was observed in the non-baroreflex $(p=0.80)$ or baroreflex $(p=0.88)$ causal events during centrifugation compared to standing (Table 3). Therefore, the observations of current study suggest centrifugation at feet is capable of evoking autonomic control of blood pressure analogous to standing.

\section{Limitations and Future Directions}

Experimental protocol in the current study was not randomized, i.e., centrifugation always followed the stand test. Although a 15-min recovery period was adopted to minimize the effects of stand test on centrifugation, in future study randomized experimental protocol should be considered. Furthermore, due to the unavailability of the respiration signal, the role of hypergravity toward the dynamics of respiration could not be studied. Respiration is known to affect both heart rate (RR) as well as blood pressure (Faes et al., 2011; Porta et al., 2012). Additionally, it may also play a role toward facilitating venous return via the physiology of respiration pump (Miller et al., 2005). Therefore, the role of respiration toward facilitating blood pressure homeostasis in response to orthostatic challenge shall be investigated in the future. Moreover, the blood volume redistribution in the splanchnic bed and the lower periphery due to standing and 2-g centrifugation shall also be measured and compared in the future. Orthostatic challenges evoked via a source that eliminates the effect of gravity such as lower-body negative pressure is observed to be different from that due to natural gravity (such as head-up tilt) (Taneja et al., 2007). Certain blood pressure regulatory controls changed only at 2-g compared to supine (Table 4), which indicate 2 -g was more stressful than standing. Accurate information regarding the degree of blood pooling achieved during each experimental condition will shed further light pertaining to the vigor of 2 -g in relation to standing.

Moreover, additional mechanisms that account for blood pressure regulation such as skeletal muscle pump (cardiopostural blood pressure regulation) shall also be investigated and compared in the future (Garg et al., 2014; Verma et al., 2017a; $\mathrm{Xu}$ et al., 2017). Furthermore, due to small sample size, the gender effect on blood pressure regulation and alteration in the dynamics of such behavior under artificial hypergravity remains to be understood. Female astronauts account for approximately $22 \%$ of total astronaut population (Harm et al., 2001), and studies have demonstrated significant gender difference in autonomic mechanisms leading to stable stance and in response to countermeasures designed to mitigate deleterious effect of spaceflight deconditioning (Arzeno et al., 2013; Goswami et al., 2015b; Hughson et al., 2016; Macaulay et al., 2016). Therefore, generalization of gender effect would further improve our understanding regarding the potential of SAHC as a training tool toward evoking blood pressure regulatory controls analogous to standing.

\section{CONCLUSION}

Cardiovascular adaptation to microgravity impairs autonomic control of blood pressure, consequently, astronauts are 
susceptible to orthostatic intolerance on return to gravitational environment. Sporadic training in artificial hypergravity is proposed to mitigate the effects of spaceflight deconditioning. SAHC is a promising tool for simulating artificial gravity of different g-loads. The response of blood pressure regulatory controls to simulated hypergravity in relation to standing is not well-established in the literature.

In the current article, we investigated the response of autonomic control of blood pressure during centrifugation (1-g and 2-g) in relation to standing. While no difference was observed in the autonomic control of blood pressure between standing and centrifugation, the blood pressure regulatory indices during standing and centrifugation (mostly 2-g) were significantly different from baseline (Table 4). The findings of the current study lead us to conclude that 2-g centrifugation at feet via SAHC has potential to evoke autonomic control of blood pressure analogous to standing, therefore, a potential training tool toward reducing

\section{REFERENCES}

Antonutto, G., and di Prampero, P. E. (2003). Cardiovascular deconditioning in microgravity: some possible countermeasures. Eur. J. Appl. Physiol. 90, 283-291. doi: 10.1007/s00421-003-0884-5

Artiles, A. D., Heldt, T., and Young, L. R. (2016). Effects of artificial gravity on the cardiovascular system: computational approach. Acta Astronaut. 126, 395-410.

Arzeno, N. M., Stenger, M. B., Lee, S. M. C., Ploutz-Snyder, R., and Platts, S. H. (2013). Sex differences in blood pressure control during $6^{\circ}$ head-down tilt bed rest. Am. J. Physiol. Heart Circ. Physiol. 304, H1114-H1123. doi: 10.1152/ ajpheart.00391.2012

Baisden, D. L., Beven, G. E., Campbell, M. R., Charles, J. B., Dervay, J. P., Foster, E., et al. (2008). Human health and performance for long-duration spaceflight. Aviat. Space Environ. Med. 79, 629-635. doi: 10.3357/ASEM.2314.2008

Bertinieri, G., Di Rienzo, M., Cavallazzi, A., Ferrari, A., Pedotti, A., and Mancia, G. (1988). Evaluation monitoring of baroreceptor reflex by blood pressure in unanesthetized cats. Am. J. Physiol. 254, H377-H383.

Blaber, A. P., Hinghofer-Szalkay, H., and Goswami, N. (2013a). Blood Volume Redistribution During Hypovolemia. Aviat. Space Environ. Med. 84, 59-64. doi: 10.3357/ASEM.3424.2013

Blaber, A. P., Yamamoto, Y., and Hughson, R. L. (1995). Methodology of spontaneous baroreflex assessed by surrogate data analysis. Am. J. Physiol. Circ. Physiol. 4, H1682-H1687.

Blaber, A. P., Zuj, K. A., and Goswami, N. (2013b). Cerebrovascular autoregulation: lessons learned from spaceflight research. Eur. J. Appl. Physiol. 113, 1909-1917. doi: 10.1007/s00421-012-2539-x

Buckey, J. C., Lane, L. D., Levine, B. D., Watenpaugh, D. E., Wright, S. J., Moore, W. E., et al. (1996). Orthostatic intolerance after spaceflight. J. Appl. Physiol. 81, $7-18$.

Bukley, A., Lawrence, D., and Clément, G. (2007). Generating artificial gravity onboard the Space Shuttle. Acta Astronaut. 60, 472-478. doi: 10.1016/j. actaastro.2006.09.013

Clément, G., and Pavy-Le Traon, A. (2004). Centrifugation as a countermeasure during actual and simulated microgravity: a review. Eur. J. Appl. Physiol. 92, 235-248. doi: 10.1007/s00421-004-1118-1

Clément, G. R., Bukley, A. P., and Paloski, W. H. (2015). Artificial gravity as a countermeasure for mitigating physiological deconditioning during longduration space missions. Front. Syst. Neurosci. 9:92. doi: 10.3389/fnsys.2015. 00092

Clément, G. R., Charles, J. B., and Paloski, W. H. (2016). Revisiting the needs for artificial gravity during deep space missions. Reach Rev. Hum. Space Explor. 1, 1-10. doi: 10.1016/j.reach.2016.01.001 orthostatic intolerance in astronauts on their return to Earth.

\section{AUTHOR CONTRIBUTIONS}

$\mathrm{NG}$ and $\mathrm{AB}$ conceived the centrifuge study and designed the experiment. AV and KT conceived the data analysis steps. MB, $\mathrm{NG}$, and $\mathrm{AB}$ acquired the data. DX preprocessed the data. AV performed the data and statistical analyses, wrote the manuscript, and created the figures and tables. AV, DX, AG, NG, AB, and KT interpreted the results. All authors contributed to manuscript editing and approved the final version for publication.

\section{ACKNOWLEDGMENTS}

The European Space Agency's CORA-GBF program supported this study.

Diaz, A., Trigg, C., and Young, L. R. (2015). Combining ergometer exercise and artificial gravity in a compact-radius centrifuge. Acta Astronaut. 113, 80-88. doi: 10.1016/j.actaastro.2015.03.034

Durand, M. T., Becari, C., Tezini, G. C., Fazan, R., Oliveira, M., Guatimosim, S., et al. (2015). Autonomic cardiocirculatory control in mice with reduced expression of the vesicular acetylcholine transporter. Am. J. Physiol. Heart Circ. Physiol. 309, H655-H662. doi: 10.1152/ajpheart.00114.2015

Evans, J. M., Stenger, M. B., Moore, F. B., Hinghofer-Szalkay, H., Rössler, A., Patwardhan, A. R., et al. (2004). Centrifuge training increases presyncopal orthostatic tolerance in ambulatory men. Aviat. Space Environ. Med. 75, $850-858$.

Faes, L., Nollo, G., and Porta, A. (2011). Information domain approach to the investigation of cardio-vascular, cardio-pulmonary, and vasculo-pulmonary causal couplings. Front. Physiol. 2:80. doi: 10.3389/fphys.2011.00080

Faes, L., Nollo, G., and Porta, A. (2013). Mechanisms of causal interaction between short-term RR interval and systolic arterial pressure oscillations during orthostatic challenge. J. Appl. Physiol. 114, 1657-1667. doi: 10.1152/ japplphysiol.01172.2012

Faes, L., Widesott, L., Del Greco, M., Antolini, R., and Nollo, G. (2006). Causal cross-spectral analysis of heart rate and blood pressure variability for describing the impairment of the cardiovascular control in neurally mediated syncope. IEEE Trans. Biomed. Eng. 53, 65-73. doi: 10.1109/TBME.2005.859788

Fontolliet, T., Pichot, V., Antonutto, G., Bonjour, J., Capelli, C., Tam, E., et al. (2015). Effects of gravitational acceleration on cardiovascular autonomic control in resting humans. Eur. J. Appl. Physiol. 115, 1417-1427. doi: 10.1007/ s00421-015-3117-9

Frett, T., Mayrhofer, M., Schwandtner, J., Anken, R., and Petrat, G. (2014). An innovative short arm centrifuge for future studies on the effects of artificial gravity on the human body. Microgravity Sci. Technol. 26, 249-255. doi: 10. 1007/s12217-014-9386-9

Garg, A., Xu, D., Laurin, A., and Blaber, A. P. (2014). Physiological interdependence of the cardiovascular and postural control systems under orthostatic stress. Am. J. Physiol. Heart Circ. Physiol. 307, H259-H264. doi: 10.1152/ajpheart.00171.2014

Goswami, N. (2017). Falls and fall-prevention in older persons: geriatrics meets spaceflight! Front. Physiol. 8:603. doi: 10.3389/fphys.2017.00603

Goswami, N., Bruner, M., Xu, D., Bareille, M. P., Beck, A., Hinghofer-Szalkay, H., et al. (2015a). Short-arm human centrifugation with $0.4 \mathrm{~g}$ at eye and $0.75 \mathrm{~g}$ at heart level provides similar cerebrovascular and cardiovascular responses to standing. Eur. J. Appl. Physiol. 115, 1569-1575. doi: 10.1007/s00421-015-3142-8 Goswami, N., Evans, J., Schneider, S., Von Der Wiesche, M., Mulder, E., Rössler, A., et al. (2015b). Effects of individualized centrifugation training on orthostatic 
tolerance in men and women. PLoS One 10:e0125780. doi: 10.1371/journal. pone. 0125780

Goswami, N., Loeppky, J. A., and Hinghofer-Szalkay, H. (2008). LBNP: past protocols and technical considerations for experimental design. Aviat. Space Environ. Med. 79, 459-471. doi: 10.3357/ASEM.2161.2008

Hallgren, E., Migeotte, P.-F., Kornilova, L., Delieİre, Q., Fransen, E., Glukhikh, D., et al. (2015). Dysfunctional vestibular system causes a blood pressure drop in astronauts returning from space. Sci. Rep. 5:17627. doi: 10.1038/srep17627

Hargens, A. R., Bhattacharya, R., and Schneider, S. M. (2013). Space physiology VI: exercise, artificial gravity, and countermeasure development for prolonged space flight. Eur. J. Appl. Physiol. 113, 2183-2192. doi: 10.1007/s00421-0122523-5

Hargens, A. R., and Richardson, S. (2009). Cardiovascular adaptations, fluid shifts, and countermeasures related to space flight. Respir. Physiol. Neurobiol. 169, 30-33. doi: 10.1016/j.resp.2009.07.005

Harm, D. L., Jennings, R. T., Meck, J. V., Powell, M. R., Putcha, L., and Sams, C. P. (2001). Invited Review: gender issues related to spaceflight: a NASA perspective. J. Appl. Physiol. 91, 2374-2383.

Harris, L. R., Jenkin, M., Jenkin, H., Zacher, J. E., and Dyde, R. T. (2017). The effect of long-term exposure to microgravity on the perception of upright. NPJ Microgravity 3:3. doi: 10.1038/s41526-016-0005-5

Heesch, C. M. (1999). Reflexes that control cardiovascular function. Adv. Physiol. Educ. 277, S234-S243.

Hughson, R. L., Robertson, A. D., Arbeille, P., Shoemaker, J. K., Rush, J. W. E., Fraser, K. S., et al. (2016). Increased postflight carotid artery stiffness and inflight insulin resistance resulting from 6-mo spaceflight in male and female astronauts. Am. J. Physiol. Heart Circ. Physiol. 310, H628-H638. doi: 10.1152/ ajpheart.00802.2015

Iwasaki, K. I., Sasaki, T., Hirayanagi, K., and Yajima, K. (2001). Usefulness of daily $+2 \mathrm{Gz}$ load as a countermeasure against physiological problems during weightlessness. Acta Astronaut. 49, 227-235.

Javorka, M., Czippelova, B., Turianikova, Z., Lazarova, Z., Tonhajzerova, I., and Faes, L. (2017). Causal analysis of short-term cardiovascular variability: statedependent contribution of feedback and feedforward mechanisms. Med. Biol. Eng. Comput. 55, 179-190. doi: 10.1007/s11517-016-1492-y

Jeong, S.-M., Hwang, G.-S., Kim, S.-O., Levine, B. D., and Zhang, R. (2013). Dynamic cerebral autoregulation after bed rest: effects of volume loading and exercise countermeasures. J. Appl. Physiol. 116, 24-31. doi: 10.1152/ japplphysiol.00710.2013

Kennel, M., and Brown, R. (1992). Determining embedding dimension for phasespace reconstruction using geometrical construction. Phys. Rev. A 45:3403.

Lambert, E., and Lambert, G. (2014). Sympathetic dysfunction in vasovagal syncope and the postural orthostatic tachycardia syndrome. Front. Physiol. 5:280. doi: 10.3389/fphys.2014.00280

Lambertz, D., Goubel, F., Kaspranski, R., and Pérot, C. (2003). Effects of long-term spaceflight on mechanical properties of muscles in humans. J. Appl. Physiol. 94, 490-498.

LeBlanc, A., Gogia, P., Schneider, V., Krebs, J., Schonfeld, E., and Evans, H. (1988). Calf muscle area and strength changes after five weeks of horizontal bed rest. Am. J. Sports Med. 16, 624-629. doi: 10.1177/036354658801600612

Lee, S. M. C., Feiveson, A. H., Stein, S., Stenger, M. B., and Platts, S. H. (2015). Orthostatic intolerance after ISS and space shuttle missions. Aerosp. Med. Hum. Perform. 86, 54-67. doi: 10.3357/AMHP.EC08.2015

Macaulay, T. R., Macias, B. R., Lee, S. M., Boda, W. L., Watenpaugh, D. E., and Hargens, A. R. (2016). Treadmill exercise within lower-body negative pressure attenuates simulated spaceflight-induced reductions of balance abilities in men but not women. NPJ Microgravity 2:16022. doi: 10.1038/npjmgrav.2016.22

Malik, M., Bigger, J., Camm, A., Kleiger, R., Malliani, A., Moss, A., et al. (1996). Heart rate variability: standards of measurement, physiological interpretation, and clinical use. Eur. Heart J. 17, 354-381.

Manzey, D. (2004). Human missions to Mars: new psychological challenges and research issues. Acta Astronaut. 55, 781-790.

Marwan, N. (2017). Cross Recurrence Plot Toolbox for MATLAB, Ver. 5.21 (R31.2). Available at: http://tocsy.pik-potsdam.de/CRPtoolbox/ [accessed June 6, 2017].

Mccracken, J. M. (2016). Exploratory Causal Analysis with Time Series Data. San Rafael, CA: Morgan \& Claypool.

Miller, J. D., Pegelow, D. F., Jacques, A. J., and Dempsey, J. A. (2005). Skeletal muscle pump versus respiratory muscle pump: modulation of venous return from the locomotor limb in humans. J. Physiol. 563, 925-943. doi: 10.1113/ jphysiol.2004.076422

Morita, H., Abe, C., and Tanaka, K. (2016). Long-term exposure to microgravity impairs vestibulo-cardiovascular reflex. Sci. Rep. 6:33405. doi: 10.1038/ srep33405

Norsk, P. (2014). Blood pressure regulation IV: adaptive responses to weightlessness. Eur. J. Appl. Physiol. 114, 481-497. doi: 10.1007/s00421013-2797-2

Olufsen, M. S., Ottesen, J. T., Tran, H. T., Ellwein, L. M., Lipsitz, L. A., and Novak, V. (2005). Blood pressure and blood flow variation during postural change from sitting to standing: model development and validation. J. Appl. Physiol. 99, 1523-1537. doi: 10.1152/japplphysiol.00177. 2005

Olufsen, M. S., Tran, H. T., and Ottesen, J. T. (2004). Modeling cerebral blood flow control during posture change from sitting to standing. Cardiovasc. Eng. 4, 47-58.

Otsuka, K., Cornelissen, G., Kubo, Y., Hayashi, M., Yamamoto, N., Shibata, K., et al. (2015). Intrinsic cardiovascular autonomic regulatory system of astronauts exposed long-term to microgravity in space: observational study. NPJ Microgravity 1:15018. doi: 10.1038/npjmgrav.2015.18

Porta, A., Bassani, T., Bari, V., Pinna, G. D., Maestri, R., Guzzetti, S., et al. (2012). Accounting for respiration is necessary to reliably infer granger causality from cardiovascular variability series. IEEE Trans. Biomed. Eng. 59, 832-841. doi: 10.1109/TBME.2011.2180379

Robertson, D. (1999). The epidemic of orthostatic tachycardia and orthostatic intolerance. Am. J. Med. Sci. 317, 75-77.

Shaw, B. H., and Claydon, V. E. (2014). The relationship between orthostatic hypotension and falling in older adults. Clin. Auton. Res. 24, 3-13. doi: 10.1007/ s10286-013-0219-5

Silva, A. S., Ariza, D., Dias, D. P. M., Crestani, C. C., and Martins-Pinge, M. C. (2015). Cardiovascular and autonomic alterations in rats with Parkinsonism induced by 6-OHDA and treated with L-DOPA. Life Sci. 127, 82-89. doi: 10. 1016/j.lfs.2015.01.032

Silvani, A., Calandra-buonaura, G., Johnson, B. D., Helmond, N., Van Barletta, G., and Cecere, A. G. (2017). Physiological mechanisms mediating the coupling between heart period and arterial pressure in response to postural changes in humans. Front. Physiol. 8:163. doi: 10.3389/fphys.2017.00163

Smith, J. J., Porth, C. M., and Erickson, M. (1994). Hemodynamic response to the upright posture. J. Clin. Pharmacol. 34, 375-386.

Stenger, M. B., Evans, J. M., Knapp, C. F., Lee, S. M. C., Phillips, T. R., Perez, S. A., et al. (2012). Artificial gravity training reduces bed rest-induced cardiovascular deconditioning. Eur. J. Appl. Physiol. 112, 605-616. doi: 10.1007/s00421-0112005-1

Stenger, M. B., Evans, J. M., Patwardhan, A. R., Moore, F. B., Hinghofer-Szalkay, H., Rössler, A., et al. (2007). Artificial gravity training improves orthostatic tolerance in ambulatory men and women. Acta Astronaut. 60, 267-272. doi: 10.1016/j.actaastro.2006.08.008

Stewart, J. M., Medow, M. S., Montgomery, L. D., and McLeod, K. (2004). Decreased skeletal muscle pump activity in patients with postural tachycardia syndrome and low peripheral blood flow. Am. J. Physiol. Heart Circ. Physiol. 286, H1216-H1222. doi: 10.1152/ajpheart.00738.2003

Sugihara, G., May, R., Ye, H., Hsieh, C. H., Deyle, E., Fogarty, M., et al. (2012). Detecting causality in complex ecosystems. Science 338, 496-500. doi: 10.1126/ science. 1227079

Svacinova, J., Javorka, M., Novakova, Z., Zavodina, E., Czippelova, B., and Honzikova, N. (2015). Development of causal interactions between systolic blood pressure and inter-beat intervals in adolescents. Physiol. Res. 64, 821-829.

Takamatsu, Y., Koike, W., Takenouchi, T., Sugama, S., Wei, J., Waragai, M., et al. (2016). Protection against neurodegenerative disease on Earth and in space. NPJ Microgravity 2:16013. doi: 10.1038/npjmgrav.2016.13

Tanaka, K., Nishimura, N., and Kawai, Y. (2016). Adaptation to microgravity, deconditioning, and countermeasures. J. Physiol. Sci. 67, 271-281. doi: 10.1007/ s12576-016-0514-8

Taneja, I., Moran, C., Medow, M. S., Glover, J. L., Montgomery, L. D., and Stewart, J. M. (2007). Differential effects of lower body negative pressure and upright tilt on splanchnic blood volume. Am. J. Physiol. Heart Circ. Physiol. 292, H1420-H1426. doi: 10.1152/ajpheart.01096.2006 
Verma, A. K., Garg, A., Xu, D., Bruner, M., Fazel-Rezai, R., Blaber, A. P., et al. (2017a). Skeletal muscle pump drives control of cardiovascular and postural systems. Sci. Rep. 7:45301. doi: 10.1038/srep45301

Verma, A. K., Xu, D., Garg, A., Cote, A. T., Goswami, N., Blaber, A. P., et al. (2017b). Non-linear heart rate and blood pressure interaction in response to lower-body negative pressure. Front. Physiol. 8:767. doi: 10.3389/fphys.2017. 00767

White, R. J., and Averner, M. (2001). Humans in space. Nature 409, 1115-1118. doi: 10.1038/35059243

Williams, D., Kuipers, A., Mukai, C., and Thirsk, R. (2016). Acclimation during space flight: effects on human physiology. Mil. Med. Res. 180, 1317-1323. doi: $10.1503 / \mathrm{cmaj} .090628$

Xu, D., Verma, A. K., Garg, A., Bruner, M., Fazel-rezai, R., Blaber, A. P., et al. (2017). Significant role of the cardiopostural interaction in blood pressure regulation during standing. Am. J. Physiol. Heart Circ. Physiol. 313, H568-H577. doi: 10.1152/ajpheart.00836.2016

Yanagida, R., Ogawa, Y., Ueda, K., Aoki, K., and Iwasaki, K. (2014). Sustained mild hypergravity reduces spontaneous cardiac baroreflex sensitivity. Auton. Neurosci. Basic Clin. 185, 123-128. doi: 10.1016/j.autneu.2014. 07.010
Zander, V., Anken, R., Pesquet, T., Brungs, S., and Latsch, J. (2013). Short radius centrifuges - a new approach for life science experiments under hyper-g conditions for applications in space and beyond. Recent Patents Space Technol. $3,74-81$.

Zhong, Y., Jan, K., Ju, K. H., and Chon, K. H. (2006). Quantifying cardiac sympathetic and parasympathetic nervous activities using principal dynamic modes analysis of heart rate variability. Am. J. Physiol. Heart Circ. Physiol. 291, H1475-H1483. doi: 10.1152/ajpheart.00005.2006

Conflict of Interest Statement: The authors declare that the research was conducted in the absence of any commercial or financial relationships that could be construed as a potential conflict of interest.

Copyright (๑) 2018 Verma, Xu, Bruner, Garg, Goswami, Blaber and Tavakolian. This is an open-access article distributed under the terms of the Creative Commons Attribution License (CC BY). The use, distribution or reproduction in other forums is permitted, provided the original author(s) and the copyright owner are credited and that the original publication in this journal is cited, in accordance with accepted academic practice. No use, distribution or reproduction is permitted which does not comply with these terms. 